\title{
Snowflake Divertor Plasmas on TCV
}

\author{
F Piras, S Coda, I Furno, J-M Moret, R A Pitts ${ }^{1}$, \\ O Sauter, B Tal'2, G Turri, A Bencze, B P Duval, F Felici, \\ A Pochelon and C Zucca \\ Ecole Polytechnique Fédérale de Lausanne (EPFL) \\ Centre de Recherches en Physique des Plasmas (CRPP) \\ Association Euratom-Confédération Suisse \\ Station 13, CH-1015 Lausanne, Switzerland \\ 1 ITER Organization, Cadarache, F-13108, St Paul-lez-Durance, France \\ 2 KFKI Research Institute for Particle and Nuclear Physics, EURATOM \\ Association \\ E-mail: francesco.piras@epfl.ch
}

\begin{abstract}
Starting from a standard single null X-point configuration, a second order null divertor (snowflake) has been successfully created on the TCV tokamak. The magnetic properties of this innovative configuration have been analyzed and compared with a standard X-point configuration. For the snowflake divertor, the connection length and the flux expansion close to the separatrix exceed those of the standard X-point by more than a factor of 2 . The magnetic shear in the plasma edge is also larger for the snowflake configuration.
\end{abstract}

PACS numbers: 28.52-s, 52.55-s, 52.55.Fa, 52.55.Rk

\section{Introduction}

In commercial fusion power plants based on the tokamak concept, the plasma is confined within closed magnetic flux surfaces generated by a combination of fields due to currents flowing in external conductors and in the plasma. The Last Closed Flux Surface (LCFS) defines the shape of the plasma cross-section and it is bounded either by the intersection of closed magnetic surfaces with a solid surface (limited plasma) or by the magnetic field itself (diverted plasma). In those magnetic confinement devices, power exhaust handling and plasma wall interaction must be mastered to a level compatible with wall materials. Different solutions have been proposed to reduce the plasma-wall interaction optimizing the divertor region by acting on the magnetic field topology [1-4]. One of these solutions is the so-called snowflake divertor $[1,2]$.

The basic concept of the snowflake divertor (SF) is illustrated in Fig. 1. In a standard X-point configuration (not shown here) the poloidal magnetic field vanishes at the null point (first order null). An SF diverted configuration is charaterized by a second order null, i.e. the first derivatives of the magnetic field also vanish at the null point and the separatrix divides the poloidal plane into six sectors, Fig. 1(b). Perturbing the exact SF configuration by shifting the plasma column away from the null point or towards it, while keeping divertor currents $I_{d 1}, I_{d 2}$ and plasma current $I_{p}$ constant, produces the magnetic configurations which are respectively shown in 


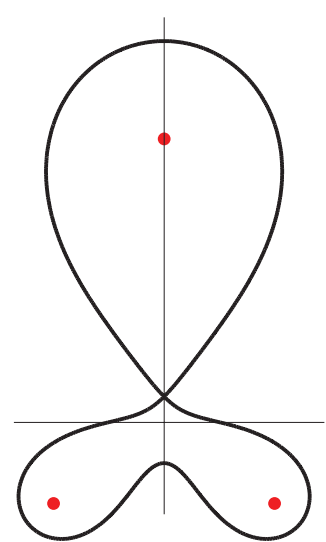

(a)

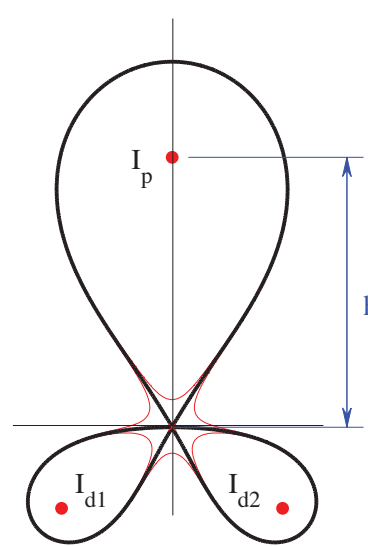

(b)

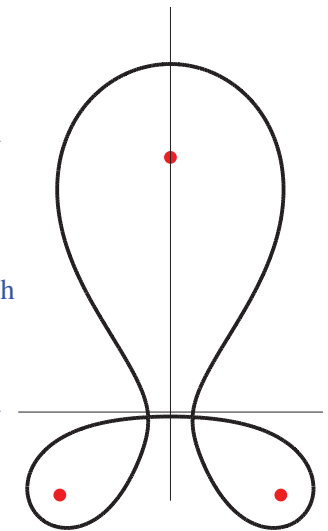

(c)

Figure 1. SF configurations using a straight tokamak model. The circles represent the current filaments (plasma, $I_{p}$, and divertor conductors, $I_{d 1,2}$ ) and the bold black line is the separatrix. The SF+ configuration in (a) and the SFconfiguration in (c) have been obtained by shifting the plasma position of the SF configuration vertically by $\pm 5 \%$, while keeping $I_{p}$ and $I_{d 1,2}$ constant.

Fig. 1(a) and Fig. 1(c). In these two configurations, the first derivatives of the poloidal magnetic field are small compared to those of a standard X-point (single null configuration, SN). Following Ref. [1, 2], we will refer to these two configurations as of snowflake-plus $(\mathrm{SF}+)$ and snowflake-minus $(\mathrm{SF}-)$ respectively.

The second-order null modifies the magnetic topology near the plasma boundary and is therefore expected to affect the edge plasma properties. In particular, the flux expansion around the the null point is 2-3 times larger than in the SN configuration and the connection length in that region increases, reducing the local heat load to the divertor plates [1, 2]. Additionally, the magnetic shear in the edge where an H-mode pedestal would lie is modified, providing a possible way to influence Edge Localized Modes (ELMs) activity [5]. Squeezing the flux tubes near the null point may also decouple the turbulence in the divertor legs and in the Scrape-Off Layer (SOL) and slow down any radial blob displacement $[6,7]$.

In this letter, we present results of the first SF diverted plasma experiments in TCV. The paper is organized as follows: in the next section, the experimental setup for TCV is presented and machine's capability to produce this configuration is investigated. Section III describes the realization of these configurations on TCV and considers the magnetic properties of the equilibrium along with a few selected experimental results. In Section IV the paper is summarized.

\section{Snowflake feasibility on TCV}

TCV (Tokamak à Configuration Variable, $R=0.88 \mathrm{~m}, a=0.25 \mathrm{~m}, I_{p} \leq 1 \mathrm{MA}$, $\kappa \leq 2.8$ and $\left.B_{\phi}=1.43 \mathrm{~T}\right)$ is constructed to explore the effects of plasma shaping with respect to stability and performance [8]. As illustrated in Fig. 2 , the machine design is up-down symmetric. The 16 independently powered poloidal shaping coils (E-F) allow the generation of a wide range of magnetic configurations. Graphite armour tiles cover $90 \%$ of the in-vessel plasma-facing surfaces. Most plasma configurations use 
the central column as a limiter or divertor surface. As a consequence, its protection tiles are designed to withstand high heat loads [9].

To assess the capability of TCV for crating an SF divertor, a limiter plasma equilibrium is modified to obtain a second order null. The initial Grad-Shafranov equilibrium is created with FBTE (Free Boundary Tokamak Equilibrium) and MGAMS (Matrix Generation Algorithm and Measurement Simulation) [10, 11], the suite of software tools used routinely on TCV to determine the poloidal coil currents for a given plasma configuration. The poloidal coil currents required to create an SF from a limited plasma equilibrium are evaluated by imposing the following conditions:

1. the magnetic field must vanish at the null point:

$$
-\mathbf{B}_{n 0}=\underline{\underline{B}}_{n c} \cdot \delta \mathbf{I}_{c}
$$

where $\mathbf{B}_{\mathbf{n} \mathbf{0}}$ is the poloidal magnetic field vector at the desired null point for the initial limited configuration, $\underline{\underline{\mathbf{B}}}_{n c}$ is the matrix containing the Green's functions used to evaluate the magnetic field at the null point from the currents in the poloidal coils and $\delta \mathbf{I}_{c}$ is the vector with the corrections in the poloidal coil currents;

2. the derivatives of the magnetic field must also vanish at the null point:

$$
-\mathbf{d B}_{\mathbf{n o}}=\underline{\underline{\mathbf{d B}}}_{n c} \cdot \delta \mathbf{I}_{c}
$$

where $\mathbf{d B}_{\mathbf{n} 0}$ is a vector containing the all derivatives of the poloidal magnetic field at the null point before the correction and $\underline{\underline{\mathbf{d B}}}_{n c}$ is a matrix containing the spatial derivatives of the Green's functions used to evaluate the derivatives of the magnetic field in the $r$ and $z$ directions at the null point from the currents in the poloidal coils;

3. the poloidal flux perturbation in the main plasma region produced by the change in the coil currents is minimized:

$$
\mathbf{0}=\underline{\underline{\mathbf{d M}}}_{n c} \cdot \delta \mathbf{I}_{c}
$$

where $\underline{\underline{\mathbf{d M}}}_{n c}$ is used to evaluate the flux variation on a certain number of points that belong to the same flux surface, Fig. 2(a). These points are chosen to be close to the LCFS but far from the null region. This supplementary condition preserves the plasma shape.

4. the solution with the minimum change of the poloidal coil currents is imposed:

$$
\min \left(\left|\delta \mathbf{I}_{c}\right|^{2}\right) \text {. }
$$

A solution to equations (1-4) is determined using a least squared approach. This minimization process is applied to produce the calculated SF configuration shown in Fig. 2(a) together with the currents in each coil, Fig. 2(b), for $I_{p}=500 \mathrm{kA}$. In Fig. 2(b), the currents in the poloidal coils for the initial limited plasma (black), a standard diverted plasma (grey) and the SF (light-grey) are compared. The data show that the currents in the poloidal coils necessary to create an SF are larger compared to an SN due to the magnetic dipole fields required by the configuration (coils E3, E4, E5 and F3, F4, F5). Nevertheless, the currents are compatible with the current limits in the TCV poloidal coils $(7.7 \mathrm{kA})$. Different SF configurations are possible within the coil current limits. We focus our attention on a configuration with positive 


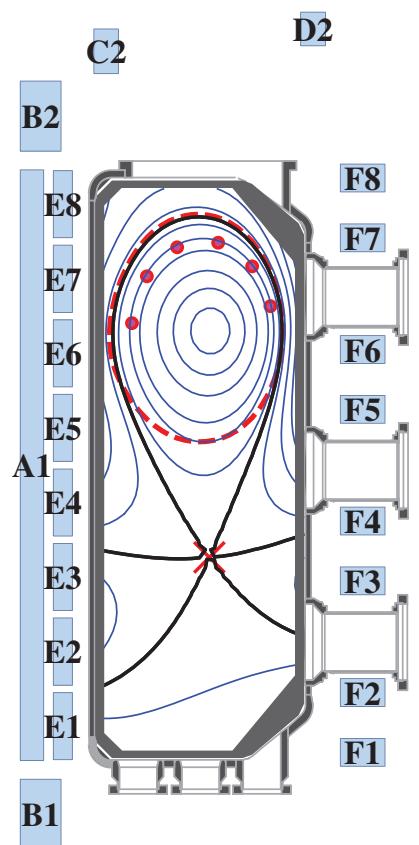

C1

(a)

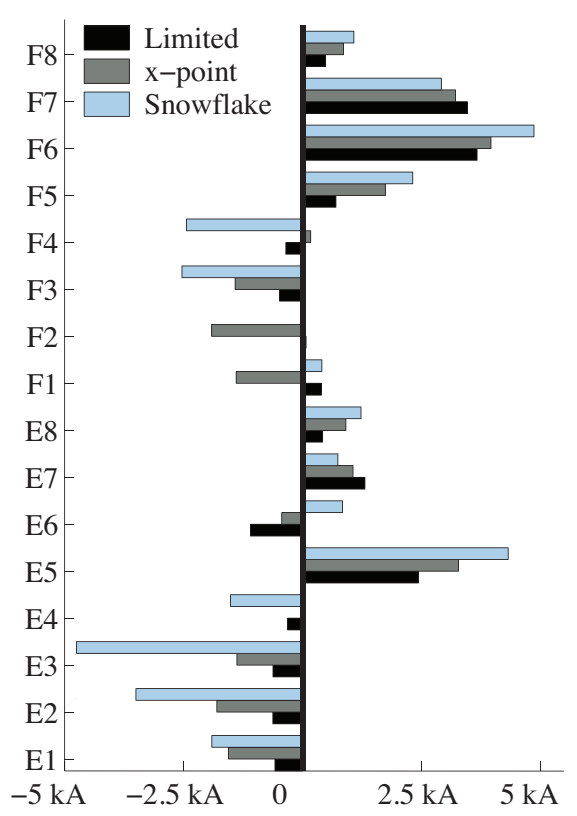

(b)

Figure 2. (a) TCV vessel and coils geometry: $\mathrm{E}$ and $\mathrm{F}$ are the 16 poloidal field coils and A,B,C and D are the ohmic coils. The results from the magnetic perturbed equilibrium approach are also shown for a plasma current of $500 \mathrm{kA}$. The thin lines are the flux contours for the SF configuration and the bold lines shown its separatrix; the dashed line is the LCFS of the initial limited plasma, the cross localizes the null point and the solid circles are the control points where the same-flux condition is imposed. In (b), the currents in the poloidal coils for a limited plasma (black), a standard diverted plasma (grey) and an SF (light-grey) are plotted. The maximum permitted current in the TCV poloidal field coils is $7.7 \mathrm{kA}$.

triangularity (which is expected to have better MHD stability [12]) and with one divertor strike point on the Low-Field Side (LFS) between the F3 and F4 coils, Fig. $2(\mathrm{a})$, to ensure that power is deposited on protection tiles.

The discharge parameters necessary to experimentally produce the SF plasma are determined from the solution of the free boundary equilibrium problem, solved using the MGAMS/FBTE code suite. Since FBTE is not able to impose a second order null condition directly, a configuration with two X-points (close to each other) is instead evaluated. The exact SF configuration is then achieved by moving the plasma position vertically during the discharge.

\section{Experimental results}

Starting from a limited plasma, an SN configuration is created with both strike points on the central column. One of the strike points is subsequently moved to the LFS producing the SF configuration. Moving the plasma vertically, the SF+ and the SF- 
(a) $\mathrm{SF}+$
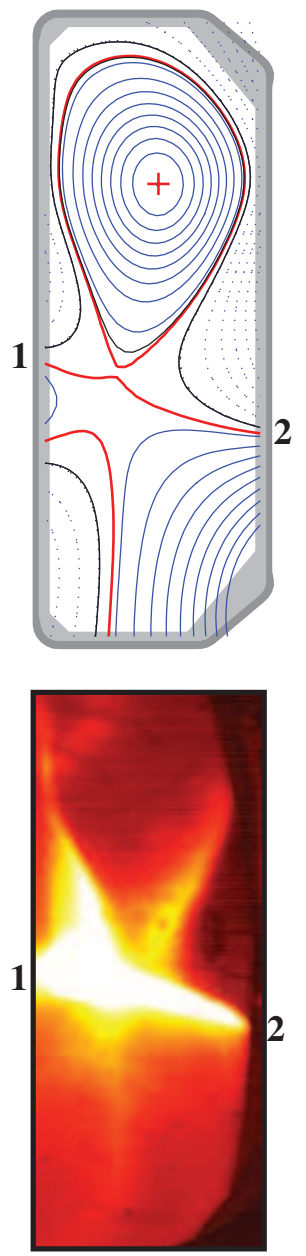

(b) $\mathrm{SF}$
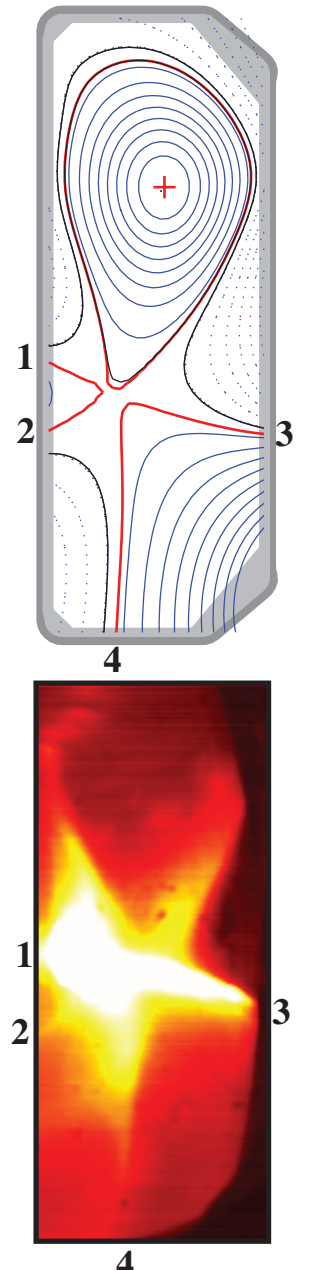

(c) SF-
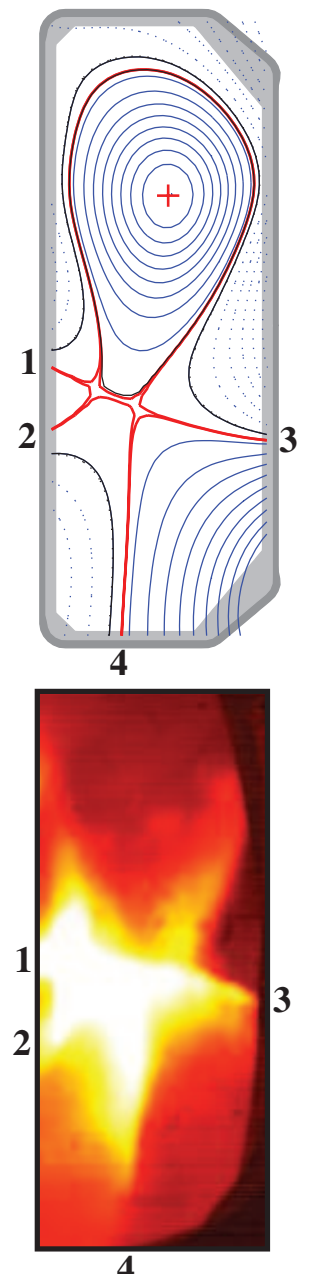

Figure 3. Equilibrium reconstructions and images from the tangential visible CCD camera for an SF+ (a), an SF (b) and an SF- configuration (c), all obtained in the same discharge by vertical plasma movement. In each frame, numbers indicate the divertor strike point positions. Notice the different vertical plasma position for each SF configuration (shot \#36151; SF+ at $0.411 \mathrm{~s}$, SF at $0.457 \mathrm{~s}, \mathrm{SF}-$ at $0.504 \mathrm{~s} ; \quad I_{p}=230 \mathrm{kA} ; \quad q_{95}=3.5 ; \quad k_{95}=1.45 ; \quad \delta_{95}=0.15$; $\left.n_{e 0}=7 \cdot 10^{19} \mathrm{~m}^{-3}\right)$.

are also established for short intervals during the same discharge. This movement is due to a slow vertical plasma position oscillation $(\sim 20 \mathrm{~Hz})$. Using the LIUQE code $[10,13]$, the magnetic equilibria for the SF configurations are reconstructed using magnetic measurements to constrain the Grad-Shafranov solution.

The reconstructed equilibria are shown in Fig. 3 together with visible light emission obtained with an unfiltered, tangentially viewing CCD camera. Although the CCD images are, unfortunately, saturated, the visible emission qualitatively confirms the presence of the SF divertor. At the relatively low plasma current and high density 


\section{(a) $\mathrm{SF}+$}

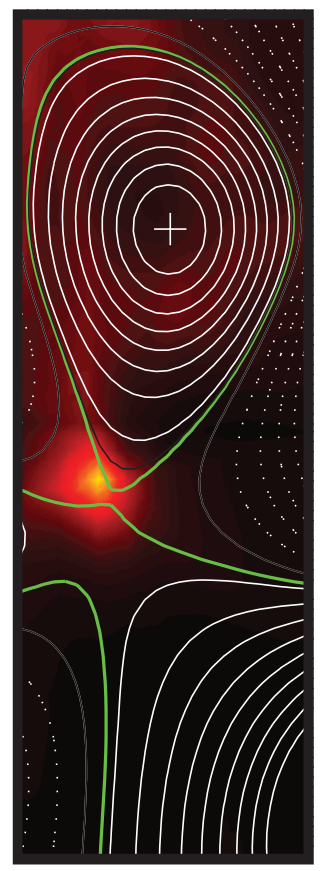

(b) SF

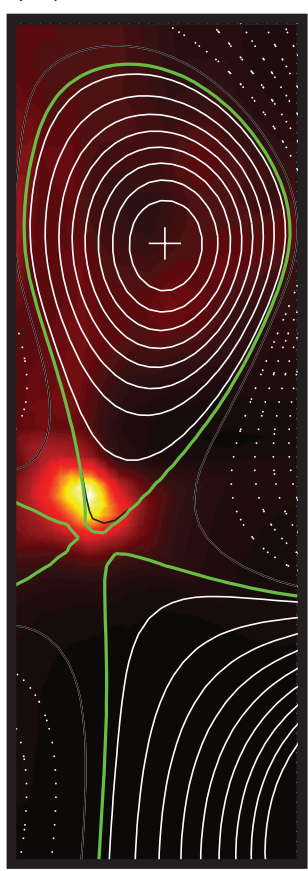

(c) SF-

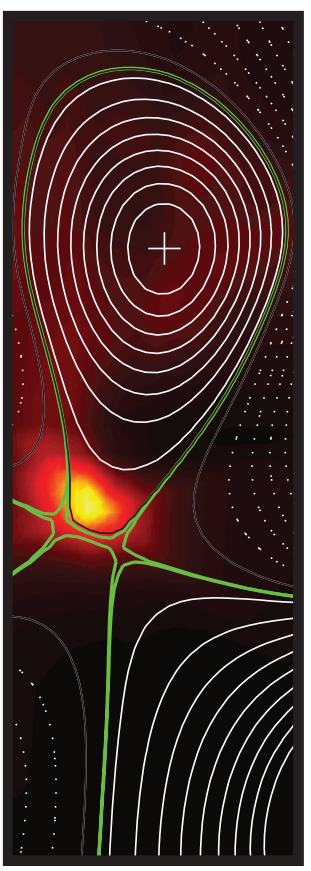

Figure 4. Tomographic reconstructions of total radiated power obtained from an array of AXUV diode pinhole cameras for the configurations in Fig. 3.

of the discharge, the majority of the emission is concentrated in the cool X-point region and along the divertor legs. Here, the plasma temperature is such as to promote strong carbon radiation from impurities generated at the graphite first wall. These visible images also show clearly that radiation occurs in all four divertors only for the SF and SF- configurations and in particular for the SF- equilibrium. Here, this is likely due to the increased size of the region over which the core plasma has direct access to the null point, increasing the power channelled through the divertor volume and thus radiated in the four divertor legs.

Fig. 4 compiles tomographic inversions of total radiation emission from a poloidal array of AXUV diode pinhole cameras [14, 15]. The inversions are performed at the same time instants as those of the CCD images in Fig. 3. In all three SF configurations, the radiation is observed to peak in the null point vicinity, with the highest levels observed for the SF equilibrium. This is again a likely consequence of the increased radiating volume in the flux expanded null point region. In a carbon dominated machine, emission at the edge is most powerful in the UV spectral region, corresponding to plasma temperatures in the range $10-20 \mathrm{eV}$. Under the conditions of these experiments, this will correspond to the X-point region, as seen in the reconstructions. The low radiation levels in the strike point vicinity, together with strong visible emission along the divertor legs, is a qualitative indication of extremely low plasma temperatures there and thus of detached divertor states. This is unsurprising given the low current and relatively high density of these plasmas, particularly when the power sharing into several divertor branches is taken into 


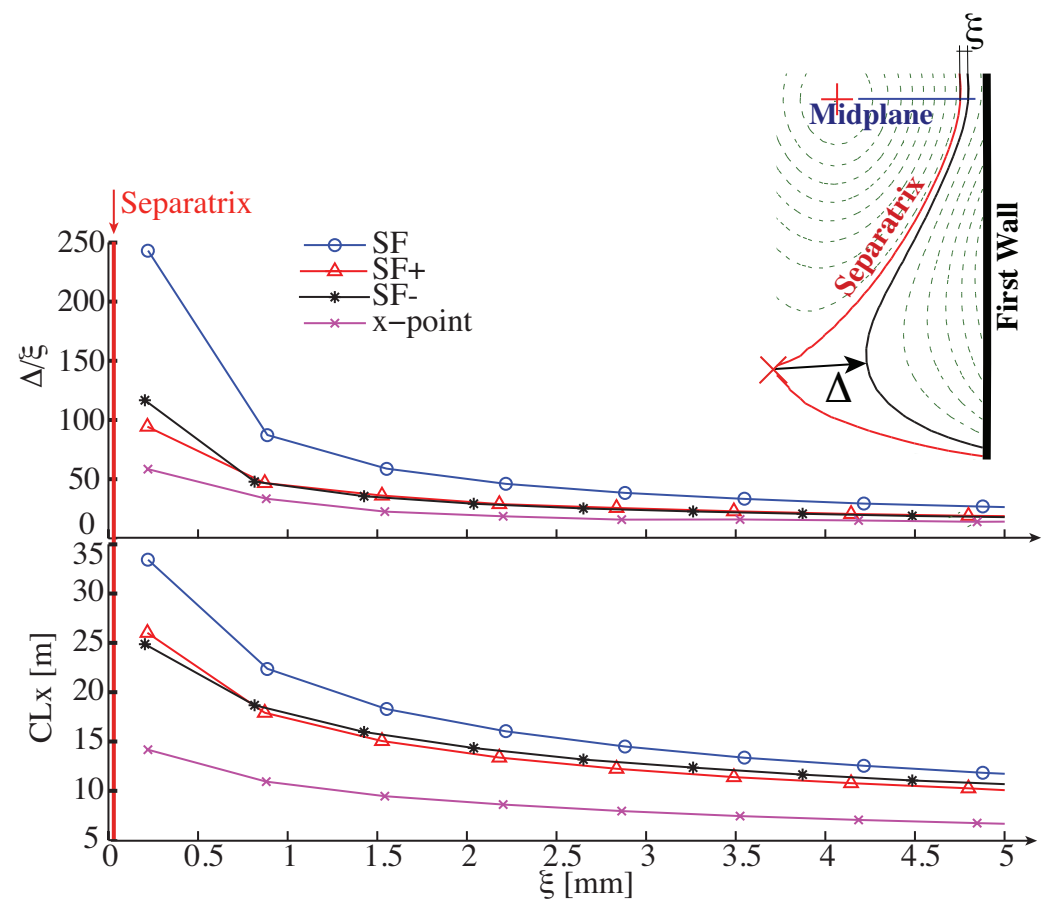

Figure 5. Flux expansion $(\Delta / \xi)$ and Connection Length (CLx) for an SF (circles), an $\mathrm{SF}+$ (triangle), an SF- (points) and an SN (crosses). The typical SOL thickness at the equatorial plane is $2 \mathrm{~cm}$. SF shot \#36151 $(\mathrm{SF}+0.411 \mathrm{~s}$, SF $0.457 \mathrm{~s}, \mathrm{SF}-0.504 \mathrm{~s})$, SN shot \#35137, $0.6 \mathrm{~s}$. In the same figure, the geometrical parameters $\Delta$ and $\xi$ are also defined.

account. The AXUV diodes have a non-linear spectral response [14, 15] and thus cannot be used to assess the absolute radiated power. The foil bolometers, unfortunately unavailable during these discharges will be used in planned future experiments.

The magnetic properties of the SF configurations are compared with those of the SN configuration using the magnetic measurements from the equilibria in Fig. 3.

For the SOL, an important parameter is the flux expansion. This quantity is related to the reduction of the poloidal magnetic field near the null point. The flux expansion influences the SOL thickness and the size of the radiating volume. Radial transport, and possibly formation of filaments in the edge/SOL region, may also be influenced by this flux expansion.

For a given flux surface, two geometrical parameters may be defined (see inset in Fig 5): $\Delta$, the minimum distance between the null-point and a flux surface in the LFS, and $\xi$, the distance between the same flux surface and the separatrix at the outer midplane. The flux expansion is now defined as the ratio $\Delta / \xi$.

In Fig. 5, the flux expansion for the SF configurations and for the SN configuration are plotted as a function of the distance $\xi$ from the separatrix. In the same figure, the connection length from the equatorial plane to the point closest to the null point (CLx) is shown as a function of $\xi$. The connection length determines the residence time of a particle in the SOL and therefore affects the radiative losses and the thermal power 


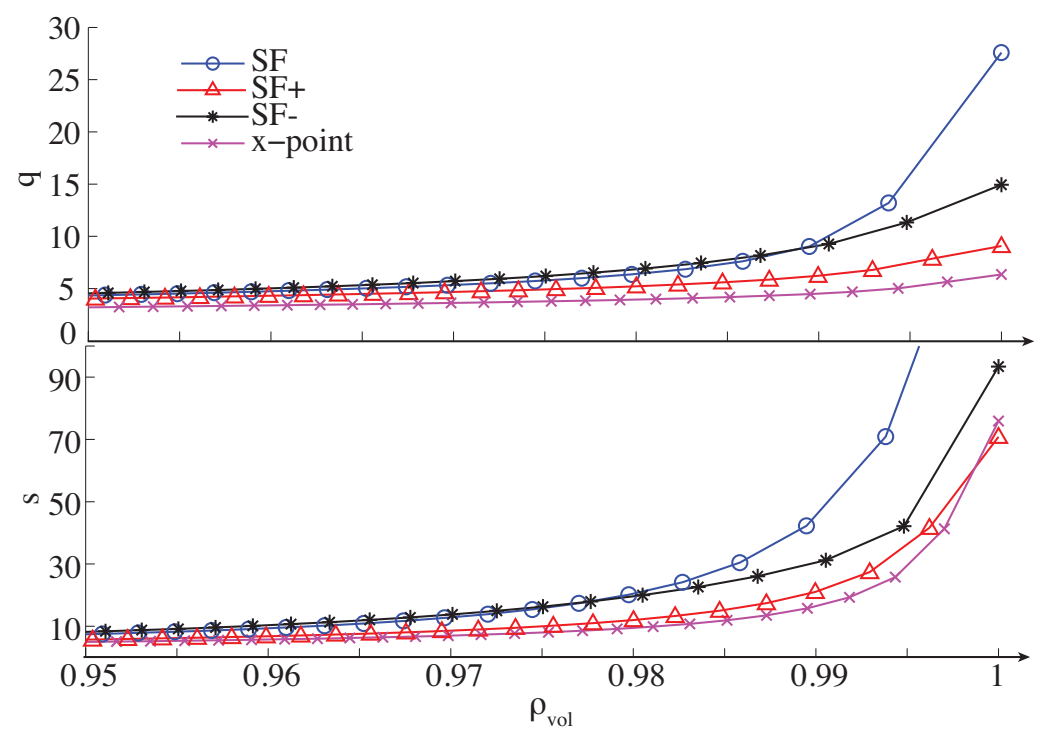

Figure 6. q-profile and magnetic shear for an SF (circles), an SF+ (triangle), an SF- (points) and an SN (crosses) as a function of $\rho_{\text {vol }}=\sqrt{V / V_{\text {edge }}}$. SF shot \#36151 (SF+ at $0.411 \mathrm{~s}, \mathrm{SF}$ at $0.457 \mathrm{~s}, \mathrm{SF}-$ at $0.504 \mathrm{~s})$, SN shot \#35137 at $0.6 \mathrm{~s}$.

to the divertor surfaces. The thickness of the SOL at the outer midplane is typically $\sim 2 \mathrm{~cm}$. The SF configuration has a flux expansion near the separatrix (Near SOL) and a connection length over twice larger than that of the SN. The SF+ and the SF- have similar values of flux expansion and connection length, with values that fall between the values computed for the SF configuration and the SN configuration.

The safety factor profile $(q)$ and the magnetic shear $\left(s=\frac{\rho_{v o l}}{q} \frac{d q}{d \rho_{v o l}}\right)$ are computed using the CHEASE code [16] and are shown in Fig. 6. The LCFS used to compute these quantities $\left(\rho_{v o l}=1\right)$ is just inside the separatrix to avoid the singularity of $q$ and $s$ at the null point. The SF configuration has a larger magnetic shear than that for the SN configuration. This difference is important for $\rho_{\text {vol }}>0.96$. The SF+ and the SF- configurations have also a larger magnetic shear compared to the SN configuration. In the case of the SF- configuration, the presence of a double null in the separatrix results in a large volume where the poloidal magnetic field is small. This property emphasizes a dissimilarity in the magnetic shear profile compared with the $\mathrm{SF}+$ configuration. Note that the profiles of the SF- are very similar to the SF up to $\rho_{v o l} \simeq 0.985$ but then become closer to the SF+ and the SN. This might lead to differences in the MHD stability limits in between the SF+ and SF- which will be investigated in the future.

\section{Summary}

This paper describes the strategy for the achievement of snowflake diverted plasmas in TCV and selected results from the first attempts. Transitions in between the three possible configurations ( $\mathrm{SF}, \mathrm{SF}+$ and $\mathrm{SF}-$ ) have been obtained by moving the plasma column vertically. The magnetic properties of the configurations have been analyzed, showing good agreement with the images from the tangential visible camera 
diagnostic and tomographically inverted total radiation emission measurements. The flux expansion, the connection length, the q-profile and the magnetic shear for the snowflake configurations have been compared with the standard X-point showing a strong variation of these parameters near the separatrix as a function of the null topology. The effects on the magnetic topology immediately inside the separatrix are not symmetric in between the SF- and the SF+ configurations. The impact with respect to edge stability for ELMs may therefore be different. This will be studied in future experimental campaigns.

The authors gratefully acknowledge contributions from M. Albergante, J. Rossel and D. D. Ryutov. This work was supported in part by the Swiss National Science Foundation.

\section{References}

[1] Ryutov D. D. et al 2008 Phys. Plasmas 15092501

[2] Ryutov D. D. et al 2007 Phys. Plasmas 14064502

[3] Kukushkin A. S. et al 2005 Nucl. Fusion 45608

[4] Mahdavi M. A. et al 1981 Phys. Rev. Lett. 471602

[5] Snyder P. B. et al 2002 Phys. Plasmas 92037

[6] Farina D. et al 1993 Nucl. Fusion 331315

[7] Cohen R. H. et al 2006 Contrib. Plasma Physics 46678

[8] Hofmann F. et al 1994 Plasma Phys. Control. Fusion 36 B277

[9] Pitts R. A. et al 1999 Nucl. Fusion 391433

[10] 1988 Hofmann F. Computer Physics Communications 48207

[11] Hofmann F. et al 1995 Proc. 22nd EPS Conf. on Controlled Fusion and Plasma Physics(Bournemouth, Great Britain, 1995)

[12] Scarabosio A. et al 2007 Plasma Phys. Control. Fusion 491041

[13] Hofmann F. et al 1988 Nucl. Fusion, 281871

[14] Degeling A. W. et al 2004 Review Scientific Instruments 754139

[15] Furno I. et al 1999 Rev. Sci. Instrum. 704552

[16] 1996 Lütjens H. et al Computer Physics Communications 97219 